\title{
On the brink of conflict: the people of South Asia deserve better
}

In this editorial (BMJ 2017;357:j1528, doi:10.1136/bmj.j1528) there are two rounding errors in the table. The 2004 population for Bangladesh should be 141 not 14.1 and for Bhutan 0.63 not 63.4. 